\title{
Functional MRI using robotic MRI compatible devices for monitoring rehabilitation from chronic stroke in the molecular medicine era (Review)
}

\author{
LOUKAS G. ASTRAKAS $^{1,2}$, SYED HASSAN ABBAS NAQVI ${ }^{1,5}$, BABAK KATEB ${ }^{4,6,7,8}$ and A. ARIA TZIKA ${ }^{1,3,4}$
}

\author{
${ }^{1}$ Department of Surgery, NMR Surgical Laboratory, Massachusetts General Hospital and Shriners Burn Institute, \\ Harvard Medical School, Boston, MA 02114, USA; ${ }^{2}$ Department of Medical Physics, University of Ioannina, Ioannina 45110, \\ Greece; ${ }^{3}$ Department of Radiology, Athinoula A. Martinos Center of Biomedical Imaging, Massachusetts General Hospital, \\ Boston, MA 02114; ${ }^{4}$ Brain Mapping Foundation, West Hollywood, CA 90046, USA; ${ }^{5}$ Dow Medical College, \\ Dow University of Health Sciences, Karachi 74200, Pakistan; ${ }^{6}$ Society for Brain Mapping and Therapeutics (SBMT), \\ West Hollywood, CA 90048; ${ }^{7}$ National Center for Nano-Bio-Electronics (NCNBE), Moffett Field, \\ CA 94035-0023; ${ }^{8}$ Department of Neurosurgery, Maxine Dunitz Neurosurgical Institute, \\ Cedars Sinai Medical Center, Los Angeles, CA 90048, USA
}

Received November 16, 2011; Accepted December 27, 2011

DOI: 10.3892/ijmm.2012.942

\begin{abstract}
The number of individuals suffering from stroke is increasing daily, and its consequences are a major contributor to invalidity in today's society. Stroke rehabilitation is relatively new, having been hampered from the longstanding view that lost functions were not recoverable. Nowadays, robotic devices, which aid by stimulating brain plasticity, can assist in restoring movement compromised by stroke-induced pathological changes in the brain which can be monitored by MRI. Multiparametric magnetic resonance imaging (MRI) of stroke patients participating in a training program with a novel Magnetic Resonance Compatible Hand-Induced Robotic Device (MR_CHIROD) could yield a promising biomarker that, ultimately, will enhance our ability to advance hand motor recovery following chronic stroke. Using state-of-the art MRI in conjunction with MR_CHIROD-assisted therapy can provide novel biomarkers for stroke patient rehabilitation extracted by a meta-analysis of data. Successful completion of such studies may provide a ground breaking method for the future evaluation of stroke rehabilitation therapies. Their results will attest to the effectiveness of using MR-compatible hand devices with MRI to provide accurate monitoring during rehabilitative therapy. Furthermore, such results may identify biomarkers of brain plasticity that can be monitored during
\end{abstract}

Correspondence to: Dr A. Aria Tzika, Department of Surgery, NMR Surgical Laboratory, Massachusetts General Hospital and Harvard Medical School, 51 Blossom Street, Room 261, Boston, MA 02114, USA

E-mail: atzika@hms.harvard.edu

Key words: stroke, stroke biomarkers, rehabilitation, functional MR imaging, robotic MRI compatible devices stroke patient rehabilitation. The potential benefit for chronic stroke patients is that rehabilitation may become possible for a longer period of time after stroke than previously thought, unveiling motor skill improvements possible even after six months due to retained brain plasticity.

\section{Contents}

1. Introduction

2. Functional neuroimaging methods for evaluating stroke

3. Functional MR imaging

4. Functional neuroimaging of cerebral reorganization in stroke recovery

5. Robotic devices for patient rehabilitation

6. The Magnetic Resonance Compatible Hand-Induced Robotic Device approach

7. Brain fMRI studies

8. Functional cortical plasticity in chronic stroke induced by hand motor training

9. Significance

10. Conclusions

\section{Introduction}

Stroke is a major cause of morbidity and invalidity in modern society. The most common form occurs when an obstructed blood vessel prevents blood flow to part of the brain, depriving cells of oxygen resulting in loss of motor control. Stroke afflicts approx. 795,000 people each year and is the most prevailing cause of severe, long term disability and the cost of their care is among the fastest-growing expenses for medicare (1).

Around $80-90 \%$ of stroke survivors exhibit motor weakness and 40-50\% experience sensory dysfunction(2). The likelihood 
of improvement after stroke varies with the nature and severity of the initial deficit. For example, 6 months after stroke about $65 \%$ of patients cannot incorporate the affected hand into their usual activities. Poor upper-extremity outcomes, defined by no hand movement or only slight finger flexion with no opening 4 weeks post-stroke, are common after a hemispheric infarction with considerable damage to the corticospinal tract (3). Stroke survivors generally achieve some degree of physical recovery within 3 months of the insult. However, only $25 \%$ achieve recovery of function to the level of everyday physical functioning seen in community-matched persons who have not had a stroke (4). In particular, cognitively impaired stroke patients experience poor recovery of activities necessary for independent daily living (5). Understandably, quality of life tends to be higher among patients with better functioning than among those with worse functioning (6).

Functional recovery after stroke can be attributed to brain plasticity, which has the amazing ability to adjust itself by forming new connections between brain neurons $(7,8)$. Both animal and human studies on stroke recovery have correlated functional reinstatement with brain reorganization (8-15). For example, intracortical micro-stimulation mapping in monkeys has demonstrated that shifts of hand representations occur following focal ischemic lesions in the sensorimotor cortex $(16,17)$. In addition, brain imaging studies in chronic stroke patients have shown that plastic changes can occur, including enhanced bilateral activation of the sensorimotor cortex, increased activity in secondary or higher order sensorimotor areas and recruitment of additional cortical areas during performance of a hand sensorimotor task (12). A clear causal link between cerebral reorganization and functional recovery has been suggested $(13,15)$ and post-stroke care with the aim of reducing long-term disability continues to advance (18). Unfortunately, objective evaluation of the specific effects of rehabilitation is technically challenging (19). Substantially more information regarding the events of post-stroke functional recovery is needed to provide a firm neurobiological foundation for evaluation strategies.

Recent studies that have examined the time course of motor recovery after stroke have found that the greatest gains in motor function occur within the first month of recovery, with some additional improvement being observed up to 6 months post-stroke $(20,21)$. Improvement, especially during the first few weeks after a stroke, is attributed to the recovery of neurotransmission in spared tissue near and remote from an infarct or hemorrhage $(22,23)$. Stroke patients then generally exhibit a plateau of functional recovery and little or no further progress is expected beyond the 6- to 12-month poststroke period. This recovery profile has traditionally been attributed to the presumption that the central nervous system has a limited capacity for reorganization and improvement in function. However, recent studies of chronic post-stroke motor impairments have shown that intensive therapeutic interventions lead to significant improvements in cortical reorganization and motor function in persons tested more than one year post-stroke (24-31). Empirical evidence suggests that a plateau in motor recovery after stroke may be related to the timing and intensity of stroke rehabilitation services. Ideally, rehabilitation should occur as soon as the diagnosis of stroke is established and the individual is clear of any life- threatening problems (32). At any time after a stroke, however, cognitive, language and motor skills may improve as a result of the cerebral processes involved in ordinary learning. This experience-induced neuroplasticity includes greater excitability and recruitment of the neurons in both hemispheres of the brain, sprouting of dendrites that communicate with other neurons and strengthening of synaptic connections. We predicate that an improved understanding of the mechanisms underlying functional recovery in chronic stroke patients will help researchers design effective neurorehabilitative strategies to further improve patients' recovery.

\section{Functional neuroimaging methods for evaluating stroke}

Brain-mapping techniques have proven to be essential in comprehending the molecular, cellular and functional mechanisms of recovery after stroke (33). The currently available non-invasive functional methods for evaluating stroke are based on hemodynamic or electrophysiological principles. Techniques based on hemodynamic principles include functional magnetic resonance imaging (fMRI), positron emission tomography (PET), single-photon emission computed tomography (SPECT) and near-infrared spectroscopy (NIRS). Electrophysiological techniques include electroencephalography (EEG), magnetoencephalography (MEG) and transcranial magnetic stimulation (TMS). Reviews of each particular technique are available in the literature (34-41) and a recent integrative review of all these techniques from the viewpoint of clinical neurophysiology was recently published (42). Importantly, only fMRI and PET allow imaging of deep brain structures such as the basal ganglia. The other technologies applied in the characterization of force control after stroke only permit characterization of the cerebral cortex and TMS can only be used to map regions capable of evoking motor responses.

Given the varying features of the functional neuroimaging methods available, a combination of two or more complementary techniques may provide greater information than any single technique. The combination of one method based on hemodynamic principles and one based on electrophysiological principles would be preferable. To this end, several investigators have used fMRI in combination with EEG (39,40,43-45). By itself, EEG is responsible for recording different brain wave frequencies and patterns (46). However, when EEG is recorded simultaneously with fMRI, artifacts arising from the MRI scanner gradients interfere with the EEG and despite technical advances (47), artifact elimination still remains problematic. Nevertheless, the simultaneous recording of EEG in the MR scanning room is especially favorable for the presurgical evaluation of patients with medically intractable partial epilepsy $(40,48)$.

\section{Functional MR imaging}

The underlying principle of fMRI is to develop images of the brain, which reflect brain tissue hemodynamics and demonstrates the concept that neuronal activation is accompanied by increased regional blood flow. A valuable advantage of fMRI is that brain anatomy and blood flow can be measured simultaneously. Spatial resolution is typically in the range of $1-5 \mathrm{~mm}$ and temporal resolution is measured in seconds. 
The fMRI techniques used for mapping brain activity are blood oxygenation level-dependent (BOLD) imaging and perfusion imaging. BOLD imaging, which is the most widely applied, uses endogenous deoxyhemoglobin as a contrast source. Through this method, neuronal activation is inferred from small, local MR signal alterations proportional to hemodynamically induced changes in net deoxyhemoglobin concentration caused by task-related increases in neuronal metabolism (49). We can observe this when cortical activation causes alterations of blood oxygenation, which create changes in microscopic susceptibility measured using $\mathrm{T} 2{ }^{*}$-weighted sequences. The application of fast MR scan techniques, such as FLASH (50-52), EPI (53-55), spiral scan (56-59) and PRESTO (60-62), for fMRI has improved temporal resolution and allowed the detection of transient changes in deoxyhemoglobin concentration following presentation of brief stimuli (63-67). An alternative, relatively new fMRI approach, i.e., resting state fMRI (rs-fMRI) may allow greater assessment of changes in organization of whole functional networks (68). On the other hand, arterial spin labeling (ASL) MRI, which permits the non-invasive quantification of regional brain tissue perfused with labeled, inflowing arterial protons (69), has been used to detect evoked changes in neuronal activity (70-73). Many researchers have compared BOLD and perfusion fMRI (74-77) and reported that BOLD imaging is generally faster, more sensitive and has better temporal resolution than perfusion imaging. On the other hand, the ASL techniques are associated with fewer vascular artifacts, more closely reflect neuronal activity, and generate data that do not require temporal autocorrelations. Recently, new sequences capable of measuring BOLD contrast and perfusion simultaneously have been developed $(78,79)$.

\section{Functional neuroimaging of cerebral reorganization in stroke recovery}

Brain imaging techniques can illustrate the plastic potential of the adult human brain in healthy subjects and in stroke patients (12). Brain function changes may occur in both the stroke and non-stroke hemisphere. Functional neuroimaging studies have shown the evolution of cerebral activity in both hemispheres as patients' skills improve with training and experience (80). Serial studies using non-invasive imaging techniques have the potential to contribute to preclinical therapy evaluation by providing insights into the timing and site of stroke-relevant changes. Several techniques, with various advantages and limitations, have been used to map changes during or after stroke recovery, including positron emission tomography (PET), fMRI and transcranial magnetic stimulation (TMS) (81). Motor and language recovery are typically assessed, likely due to the ease with which they can be measured. Seminal studies from the laboratory of Richard Frackowiak (82-84) and subsequent results reported by others (85-93) have provided insights into brain reorganization following brain injury. In addition to the above techniques, single-photon emission computed tomography, near infrared spectroscopy, high-resolution electroencephalography (EEG) and magnetoencephalography have recently begun to be applied in the clinic for stroke patient evaluation. Moreover, there is reported evidence of structural plasticity co-localized with areas exhi- biting functional plasticity in the human brain after stroke (94), and there has been recently provided direct evidence of brain plasticity in chronic stroke patients $(95,96)$. Since there is limited knowledge explaining why some patients recover relatively completely, while others do not, it has been suggested that insights from functional neuroimaging studies may improve our ability to predict recovery and guide selection of individuals likely to benefit from particular treatment courses (97). Eliassen and colleagues have provided a recent review of functional neuroimaging post-stroke recovery (33).

The increased spatial resolution of fMRI has clarified neuroanatomical relationships that appeared equivocal with other methods (98). Cao et al (99) first applied fMRI to stroke recovery in a study of teenage patients who had suffered perinatal infarcts. Several stroke recovery studies have demonstrated that fMRI can, with high sensitivity, assess cortical and subcortical reorganization after stroke $(84,98,100-107)$. Widespread early activation responses in the unaffected hemisphere after stroke do not appear to be directly associated with functional recovery $(13,15,107)$ and restored function may more directly correlate with gradual reinstatement of representational neuronal fields and/or recruitment of perilesional networks $(13,15)$. Johansen-Berg et al demonstrated activation of cortical motor areas in the unaffected hemisphere during movement of paretic hands and suggested that this activation reflects an adaptive response to stroke injury in associated brain (107). These findings of biphasic cerebral rearrangements underlying functional reinstatement provide potential insights into the neurophysiological mechanisms activated by neurorehabilitative therapies, and could contribute to optimizing existing therapeutic approaches.

General conclusions regarding the influence of interventions on activation patterns are similar for both upper and lower extremity (gait) movement (though these two categories of movement are quite distinct). The specific regions identified with each type of movement differ because of the differing motor task properties and imaging modalities used in the assessment of each. Five studies examining repetitive task rehabilitation interventions have demonstrated changes in brain activation post-intervention that were associated with improved upper limb (108) or lower limb (109-111) motor performance after stroke. Some discrepancies in the brain activation patterns observed using the same intervention were apparent. Specifically, Dong et al (108) showed reduction in activation of the undamaged contralesional hemisphere after a constraint-induced movement therapy (CIMT) intervention, whereas Kopp et al (112) found that the contralesional hemisphere was recruited more after a CIMT intervention. Because the sample sizes were small in both studies, it is difficult to determine whether these differences are due to stroke severity, stroke chronicity, lesion location or some other combination of factors. Moreover, different imaging modalities were used in these two studies (EEG vs. fMRI), making a direct comparison of results strained, if not impossible. Generally, interpretation of fMRI in stroke patients is an arduous task. Particular consideration should be given to baseline circulatory status. For example, major decreases in BOLD signal have been demonstrated in stroke patients as a result of decreases in cerebral blood oxygenation during neuronal activation, commensurate to the degree of ischemia (113). In addition, 
extra- or intracranial artery diseases influence negatively the neurovascular coupling and the cerebrovascular reserve capacity and consequently decrease the BOLD signal (114).

The ability to determine how patterns of brain activation shift with improved motor performance has great implications for ongoing research informing the development of therapies intended to manipulate brain reorganization. For example, repetitive TMS applied to the cortex is being examined as a tool to promote cortical plasticity in individuals who have suffered strokes (115) and could be used with other rehabilitation therapies to further promote functional motor programs (116). In addition, as has been shown in CIMT studies $(108,112)$, that consideration of whether and how interventions shift activation in brain regions associated with the control of force will be critical for determining the efficacy of new treatment approaches. However, it appears that some degree of sparing of primary motor areas and associated network of secondary regions is a prerequisite for these types of interventions to produce functional movement and result in treatment success. Thus, the use of fMRI and other non-invasive neuroimaging techniques to identify residual anatomical areas and their relative contribution to functional movement may aid in determining which persons with stroke will benefit greatest from such treatments.

\section{Robotic devices for patient rehabilitation}

Rehabilitation is an active and dynamic process that assists a disabled person to gain the required knowledge and skills to maximize their own physical, mental and social function (117). During recent years rehabilitation robotics has emerged as a highly active collaborative research area between the robotics and medical rehabilitation communities (28,118-122). Robots provide both movement controllability and measurement reliability; making them ideal instruments to help neurologists and therapists address the challenges facing neurorehabilitation (123). Recent technological advances have made it possible to safely employ robotic devices in the intensive rehabilitative therapy of individuals with mild to severe motor impairments following neurological injury (124). Improved technology has allowed advancements that will enable these future versions of these devices to apply tension resisting the motion of joints. In addition to providing new options for treatment, this technology may enhance our understanding of the mechanisms that underlie the recovery of motor function and neural reorganization after stroke. Robot-assisted therapy has been shown to benefit patients during neurological recovery $(30,125-134)$. Specifically, persons who received robotic therapy exhibited improved gain in motor coordination and muscle strength of the exercised shoulder and elbow relative to control subjects (135). Furthermore, Volpe et al (127) reported that these improvements were sustained over a three-year period following inpatient discharge from the hospital. Although some concerns remain, most physicians show interest in purchasing robotic devices; however the biggest challenge for robotics in rehabilitation is the limited amount of scientific evidence elucidating appropriate therapy for motor dysfunction after a stroke (136). One suggestion is that therapy is more effective when it involves several functional tasks assisted by a virtual reality environment (137). Indeed robotics can be combined with virtual reality for motor rehabilitation (137).
Most MR-compatible devices are sensing systems, such as those that quantify force exerted by subjects using their hands or wrists (138-140). For example, the device by Riener et al (141) uses optical force sensors to measure handgrip strength. These systems are not capable of applying any force and are used only for monitoring. The number of actuated robotic systems for MRI is small $(142,143)$ and most of these systems have a very low range of force or motion.

Post-stroke therapy can significantly improve recovery and reduce long-term disability (18), but objective methods for evaluating the specific effects of rehabilitation are needed. While the findings of many studies have supported the hypothesis that functional brain changes accompany therapy-mediated improvements in motor skills $(14,27,93,106,112,144)$, the spatial specificity of current evaluation methods is inadequate to allow clear neuroanatomical localization of functional changes. Initial clinical studies of robotic therapy suggest that robotic devices not only improve motor recovery, but are also at least as effective as training with physical therapists alone to restore arm function and gait. While investigators have not yet conclusively demonstrated that motorized therapy is superior to comparable non-motorized therapy, the incremental improvements in clinical scales following intensive robotic therapy, although small, are statistically significant and certainly meaningful to patients.

\section{The Magnetic Resonance Compatible Hand-Induced Robotic Device approach}

Recently an electrorheological fluid (ERF)-based actuated robotic system for MRI (MR_CHIROD) has been built and tested it in adult volunteers and stroke patients for accurate, sensitive and specific information about the effectiveness of rehabilitation therapy beyond the traditional paradigms (145-148). MR_CHIROD is unique in its ability to apply resistive force to the hand over a large dynamic range (up to $200 \mathrm{~N}$ ) and monitor applied force, as well as in its physical characteristics of being compact and light.

MR_CHIROD fills the existing need for available rehabilitation robotic device that can be used with an MR scanner to measure both force and torque while applying computercontrolled time-varying force and torque. MR_CHIROD is novel in that it employs an unconventional type of semi-active actuation, namely electrorheostatical fluids (ERFs) consisting of dielectric microspheres dispersed in an insulating liquid, that make it MR-compatible (149). Although MR_CHIROD is small, light and inexpensive, it is capable of producing large, computer-controlled, time-varying resistive torque. Unlike previously described devices $(124,150)$, MR_CHIROD is the first ERF-based device that has been demonstrated to function in conjunction with fMRI for online brain mapping in chronic stroke patients $(95,149)$. Importantly, MR_CHIROD is capable of limiting and controlling a number of factors that affect its operation, making it particularly useful for homebased training given the low level of expert clinical support in the home environment which can be accompanied by low extrinsic motivation. Relative to physical therapist-facilitated training in a hospitals, home training is less expensive and more convenient, making it easier for patients to adhere to daily therapy. MR_CHIROD can be re-engineered to improve 
the cost-to-benefit ratio and therapy effectiveness by providing autonomous and recordable training programs with extrinsic motivation through virtual reality technology. Virtual reality can engage patients, increase their attention during the task and improve motivation, thus increasing the effectiveness of rehabilitation (151).

The rationale for building and using an MR-compatible hand robot is that while robotic therapy has been shown to improve arm motor function after stroke with few exceptions $(119,152)$, these efforts have not been focused on the hand (135). Given the central role that hand movements normally play in people's daily lives, more attention should be devoted to the study of rehabilitation of hand motor function after stroke. Since a major issue in hand motor therapy is how to best restore function, interventions emphasizing intense, active, repetitive movement are of high value. These interventions increase strength, accuracy and functional use when applied to subjects with paresis due to stroke. For chronic stroke patients who are in advanced stages of therapy, rehabilitation should be aimed at returning an individual to normal activities and thus incorporate resistance exercises intended to support renewed development of muscle strength. MR_CHIROD approach provides such therapy and it was motivated first by the paucity of efforts that have been made thus far concerning robotic developments for the hand and second by the unique combination of features that make our MR-compatible hand robot hold promise for enhancing traditional post-stroke therapy. These features include: i) it can provide therapy via exercises and control of the hand for long time periods in a consistent and precise manner; ii) it can be programmed for real-time adjustments of the applied force and motion according to the desired force of contraction; iii) it can measure and record performance parameters through a computer; and iv) it can be adjusted to perform with only remote human control. The latter feature extends the promise of controlling the hand robots in the MRI suite from a distance without the operator having to interrupt MRI scanning, which would improve data quality. Finally, the most notable difference between MR_CHIROD and the new breed of rehabilitation devices in use is its highly adaptive, versatile and reprogrammable nature. Computer control is intrinsic to our design and is a central theme behind this robot's operation, making it a highly effective tool. Thus, the advantages of our advanced MR-compatible hand robot are low-cost, portability, real-time abilities and versatility.

After the first generation of MR_CHIROD (145-148) two new designs have been developed; one with rotary damper/ brakes and another with linear damper/brakes. The linear damper/brake version was chosen for fabrication because of its simplicity and lower cost (153). The ERF properties and performance of the sensors were not affected by introduction of the MR_CHIROD in the scanner. Conversely, the MR images (phantom, human) suffered no degradation by the introduction of the MR_CHIROD in the MR scanner. The design and testing of the second generation MR_CHIROD was also been published (148).

An assembled MR_CHIROD is shown in Fig. 1. The MR_CHIROD consists of three major subsystems: i) an ERF resistive element, ii) handles and iii) two sensors, include an optical encoder to measure patient-induced motion and a force sensor. Each subsystem includes several components of
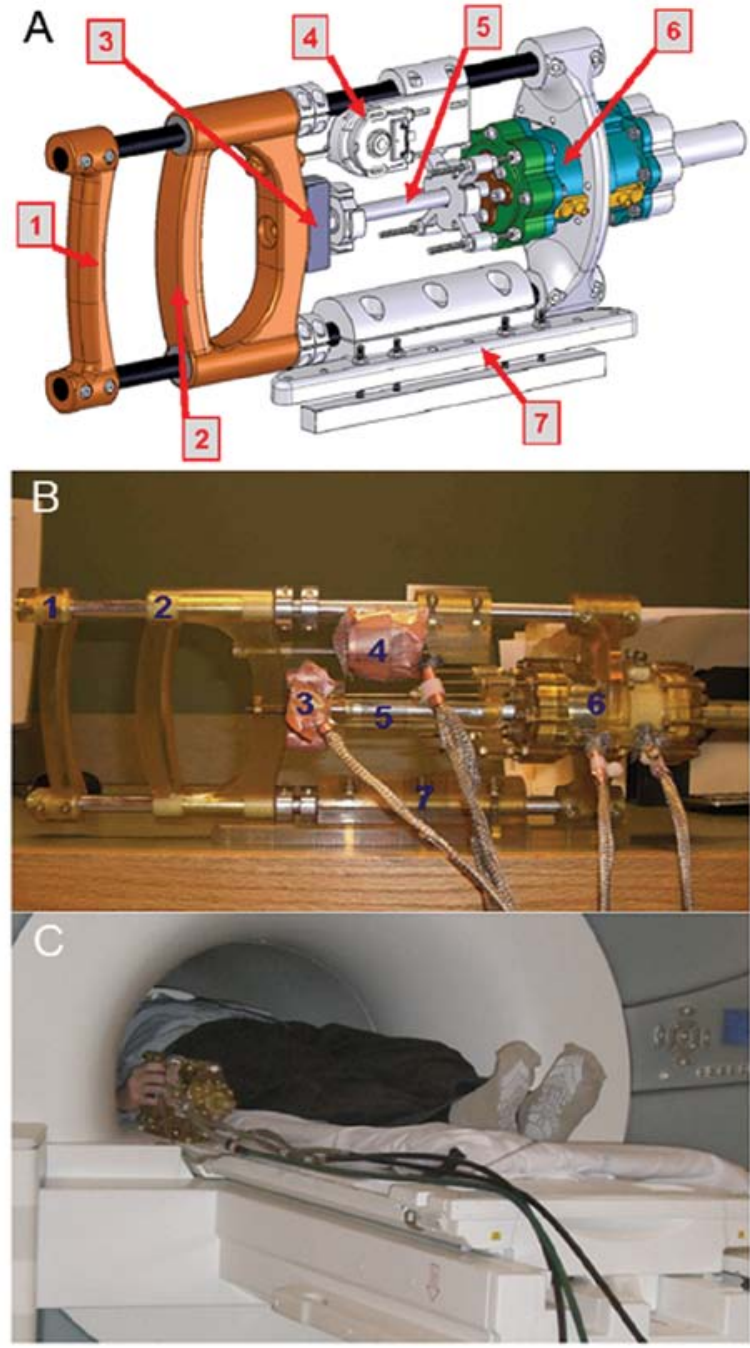

Figure 1. (A) CAD drawing; second-generation MR_CHIROD with linear exercise motion and linear damper. (1) Fixed handle; (2) Moving handle with adjustable range of motion; (3) Force sensor; (4) Position encoder; (5) Piston, connected to moving handle and to damper in ERF fluid; (6) Casing containing the ERF fluid. (7) Base allows firm attachment to the side of the magnet bed and tilt with respect to the vertical axis, maximizing ease of operation. (B) Assembled operational device; (C) MR_CHIROD attached to the scanner while a subject, lying in the magnet, operates it.

varying complexity. All components were optimally designed with strength and safety in mind for MR-compatibility and for regular and high-stress testing. The MR_CHIROD is configured to securely attach to the scanner table next to the subject who thus feels no weight. The MR_CHIROD is designed to provide up to $200 \mathrm{~N}$ resistive force and to be controlled in realtime (148). Fig. 2 summarizes the online brain MRI concept (95) The MR_CHIROD attaches securely to the scanner table next to the participant, who feels no weight.

\section{Brain fMRI studies}

Relevant published studies (13-15,30,94-96,145-149,153-162) conclude that: i) when used in conjunction with the MR CHIROD, fMRI data can provide a biomarker for neurological recovery after a stroke; ii) post-stroke cortical and sub cortical reorganization can be assessed with high sensitivity by fMRI; 


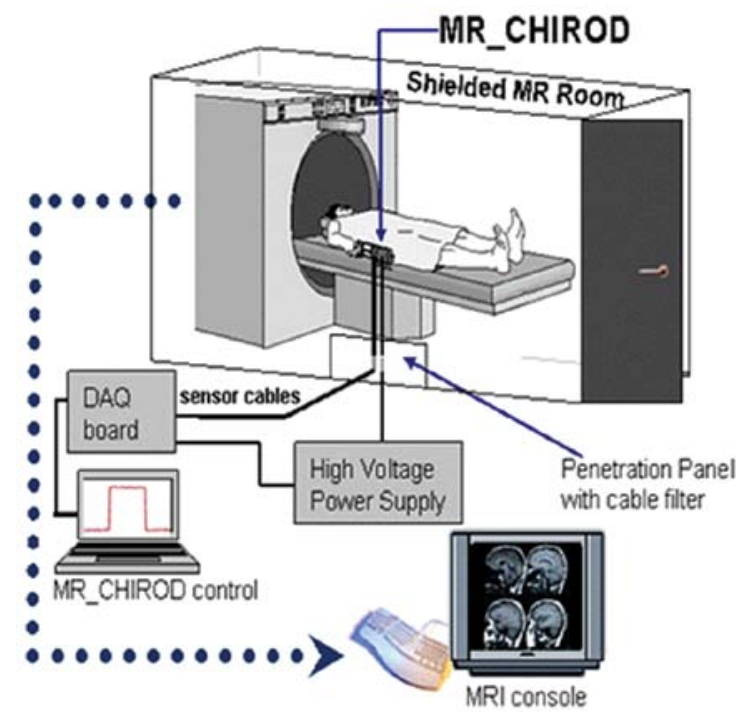

Figure 2. The Magnetic Resonance Compatible Hand-Induced Robotic Device (MR_CHIROD) concept for online brain MRI. The current MR_CHIROD (version 2) is controlled by a data acquisition (DAQ) card and DAQ software on a PC located in the operator room, outside the RF-shielded MR system. This DAQ/PC configuration can be re-engineered into a compact electronic unit. The $\mathrm{PC}$ via its $\mathrm{A} / \mathrm{D}$ and $\mathrm{D} / \mathrm{A}$ boards collects, stores and visualizes in realtime the encoder and torque measurements. Based on the selected exercise protocol, it also sends the required control voltage to the MR_CHIROD actuators. The PC voltage output is amplified using a very fast, high-voltage power supply and a high-voltage, low-current amplifier circuit board provided by the ERF manufacturer. The damper consists of two electrodes and contains the ERF fluid. The piston (piston shaft drawn in Fig. 1) moves through the ERF fluid with a controlled force of contraction provided by the voltage-controlled variable viscosity of the ERF fluid. A Faraday cage encloses the core of the device, allowing a necessary opening for the movable piston shaft. The negative electrode of the damper (connecting to the negative terminal of the power supply) and the Faraday cage are grounded to the penetration panel of the MR room. A low-pass filter (LPF) is attached to the penetration panel. Sensor readings (force, position) are transmitted through the penetration panel via grounded DSub-9 connectors. The sensor wires are coaxially shielded and grounded to the penetration panel. The sensor readings are used for real-time, closed-loop control of the ERF resistive element. The output from the control loop regulates the voltage output of the power supply, in turn ensuring control of the ERF resistive element and force of contraction.
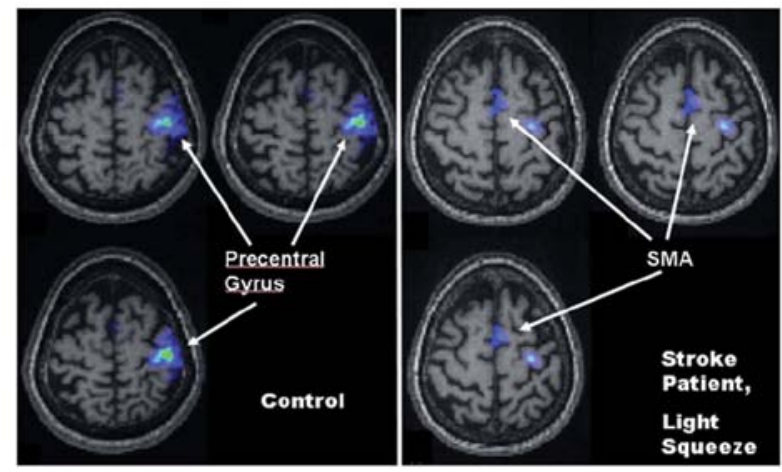

Figure 3. Activation pattern of a 75 -year-old stroke patient squeezing at $15 \%$ grip strength (right panel) compared to healthy controls (left panel). FMRI activation is superimposed on T1-weighted images. SMC activation is shown at the precentral gyrus; SMA, supplementary motor area.

iii) limb dysfunction is related to loss of brain activation; iv) functional recovery after stroke is associated with preservation

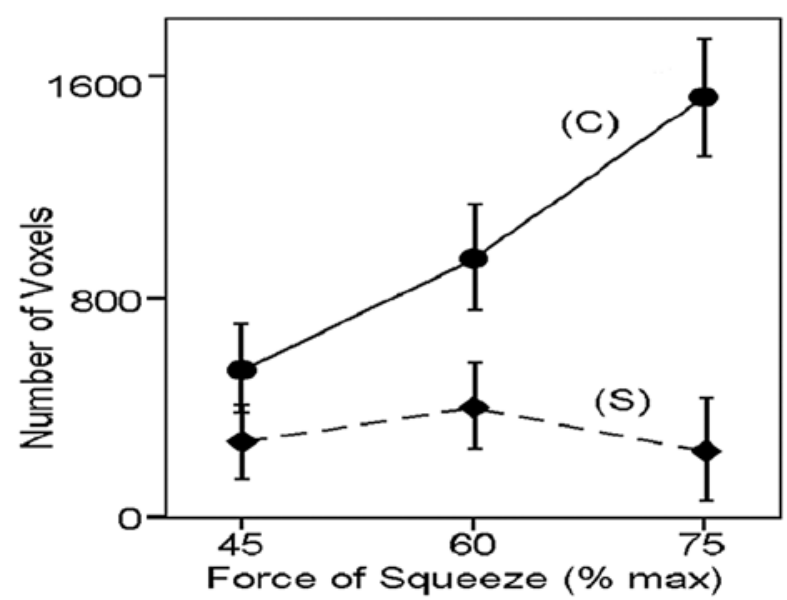

Figure 4. Activated voxels were enumerated for healthy volunteers (controls, C) and stroke patients (S) in the contralateral SMC and increased with force of squeezing in controls. Values are means \pm SE. The level of performance was consistently lower in stroke patients than in controls.

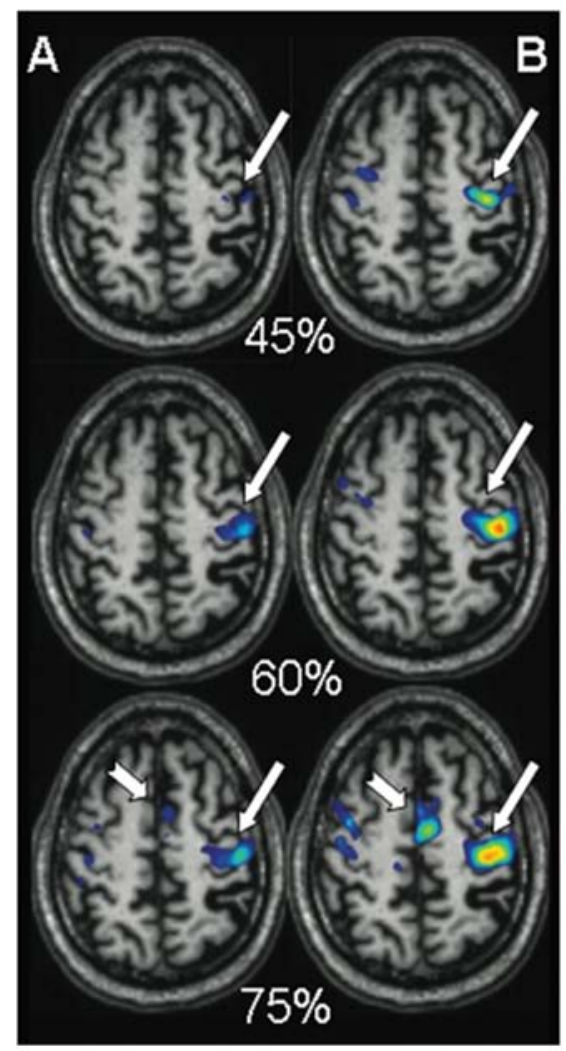

Figure 5. fMRI conducted with MR_CHIROD shows functional cortical plasticity in a chronic stroke patient. (A) Patient performance halfway through training. (B) Patient performance after full 8 -wk training. Patient squeezed the MR_CHIROD at 45, 60 and $75 \%$ maximum grip force. Activation threshold $\mathrm{P}<0.05$ corrected; activation maps are superimposed on the patient's T1-weighted anatomical images. SMC activation, longer arrow; SMA activation, shorter arrow). Red color (t-score $=10, \mathrm{P}<0.0001)$; Blue color $(\mathrm{t}$-score $=4.8, \mathrm{P}=0.05)$.

or restoration of brain activation; v) stroke affects neural connectivity; vi) goal-directed robotic therapy can improve motor abilities in stroke patients and such changes are sustained for at 


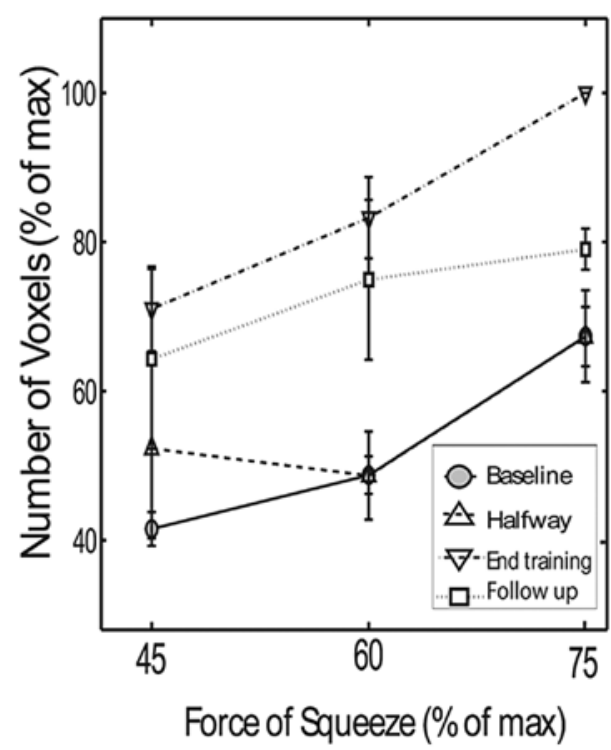

Figure 6. Number of activated voxels in the contralateral SMC as a function of squeezing force in chronic stroke patients. Online mapping was performed at four time-points: baseline (solid line); halfway through training (lower dashed line); at the end of the training (upper dashed line) and 4 weeks after training (dotted line). Note the persistence of increased cortical activation observed during and after the training period.

least four months; vii) hand training in chronic stroke patients enhances cortical activation as assessed by fMRI and improves motor performance in a manner consistent with functional plasticity; viii) structural plasticity co-localizes with regions of functional plasticity in the human brain after stroke; and ix) connectivity alterations in motor-related areas are suggestive of functional motor systems reorganization in stroke patients. Although supported by our preliminary data, the last three statements require further validation from studies with large number of participants.

Fig. 3 shows data from a 75-year-old stroke patient (2 years after a stroke that affected the left hemisphere). With a $15 \%$ effort level squeeze, both SMC and SMA were activated, but the activation in SMC (at the precentral gyrus) was less than in controls. Fig. 4 shows that the canonical curve defining the relationship between brain cortical activation and force of squeezing in controls (C) differs from the shape of the analogous curve in stroke patients (S), which is almost flat. Finally, recently published work suggests that stroke patients exhibit structural plasticity in the same sensorimotor cortical areas that exhibit functional plasticity (94). These results provide the first evidence of structural plasticity co-localized with areas exhibiting functional plasticity in the human brain after stroke.

\section{Functional cortical plasticity in chronic stroke induced by hand motor training.}

In a recent study, stroke patients training at home with exercise gel balls (Cando gel hand exercise balls; www.bpp2. com/physical_therapy_products/2932.html) underwent fMRI (95) using the second-prototype MR_CHIROD. Results are shown for a representative patient (63-year-old, right-handed male with subcortical MCA stroke, 4 years post-stroke). The number of activated voxels had increased overall and as a function of effort level at completion of the 8-week training period (Fig. 5). Fig. 6 summarizes results from 5 patients squeezing at three performance levels and over four time-points (baseline, halfway through training, end of training, and at follow-up 4 weeks after completion of training). There were a higher number of activated voxels upon completion of training than at baseline or halfway through training for all three submaximal performance levels. For example, squeezing at $60 \%$ effort at the completion of training resulted in $83.25 \pm 5.45 \%$ activated voxels, compared with $48.74 \pm 2.53 \%$ at baseline $(\mathrm{P}<0.0001)$. Significant behavioral gains were also found at the end of treatment. For example, mean arm motor Fugl-Meyer score at the end of treatment increased from $42 \pm 7$ at baseline to $55 \pm 6$ after treatment $(\mathrm{P}<0.05)$. Likewise, mean Action Research Arm Test score increased from $37 \pm 15$ at baseline to $40 \pm 14$ after treatment $(\mathrm{P}<0.05)$. SMC activation with $60 \%$ effort squeezing 4 weeks after training completion remained higher $(74.94 \pm 10.71 \%)$ than at baseline $(\mathrm{P}<0.05)$ (Fig. 6). A similar trend was observed with $75 \%$ effort squeezing, though the comparison to baseline data did not reach statistical significance. These results suggest that the increased SMC activation persists at a reduced degree 4 weeks after training. Importantly, these data demonstrate functional cortical plasticity in chronic stroke accompanied by recovery of motor performance. They also confirm a previous report by Fasoli and colleagues (30) in which chronic stroke patients subjected to goal-directed robotic therapy showed significantly improved motor abilities assessed by traditional motor evaluation; these improvements were sustained 4 months after discharge.

\section{Significance}

Assessing neuroplasticity by means of multiparametric MRI is important for the evaluation of sensorimotor brain networks. Neurological deficit may be better predicted and more precisely characterized by incorporating functional maps of injury assessed with MRI (163). Functional maps can provide insight as to which parts of a system are still functioning, thereby potentially providing new information not be evident from clinical observations alone (97).

Currently, sparse data indicate a relationship between brain activation and functional improvement during therapy suggesting that serial fMRI can be used in predicting the success and optimal duration of therapeutic intervention (108). New studies are required to fully establish the link between changes in fMRI responses and functional outcomes. Then multiparametric MRI combined with a MR-compatible robot would help neurologist to select the most appropriate rehabilitation approach and then fine-tune it based on brain activity. This approach would allow identification of the brain areas that need to be targeted in each individual patient. For example, since the integrity of the corticospinal tract (CST) is a major determinant of motor recovery (164-166), particularly within the first few weeks of recovery (167) and both the extent of structural damage to the CST and subsequent functional reorganization can be observed by MRI (168) the ipsilesional motor cortex might be targeted in patients with largely undamaged ipsilesional motor cortex and CST. In 
contrast, the contralesional motor cortex might be targeted for therapy in patients with damaged ipsilesional motor cortex and corticospinal tract. Different rehabilitation approaches would likely have differential impacts on the activity of brain areas or networks that mediate spontaneous and compensatory motor recovery in stroke patients. Task-specific training might promote spontaneous motor recovery by normalizing ipsilesional motor cortex activity. In contrast, constraint-induced movement therapy might promote an increase in recruitment of an attentional network.

\section{Conclusions}

The benefit of technology to aid in rehabilitation techniques is now begging to be realized. The Longitudinal multiparametric MRI assessment of brain reorganization in conjunction with MR-compatible robotic devices in chronic stroke is a rapidly evolving field which demonstrates outstanding potential. Stroke rehabilitation is relatively new, having been set back by the longstanding view that lost functions were not recoverable. Robotic devices similar to MR_CHIROD may prove to be valuable in restoring motor performance. The role of robotic therapy is likely to increase as the discrete components of therapeutic intervention become better-understood and robotic assessment techniques are developed in the molecular medicine era.

\section{Acknowledgements}

This study was supported in part by a grant from the National Institute of Biomedical Imaging and Bioengineering of the National Institutes of Health (Grant no. R21 EB004665-01A2) to T.A.A. We thank Dr C. Mavroidis the principal investigator of the subcontract to Northeastern University to build the MR_CHIROD. We thank Ann Power Smith, Ph.D. of Write Science Right for editorial assistance. Finally, we thank the Brain Mapping Foundation and Society for Brain Mapping and Therapeutics (SBMT) for their contributions to this research.

\section{References}

1. Dobkin BH: Clinical practice. Rehabilitation after stroke. N Engl J Med 352: 1677-1684, 2005.

2. Bogousslavsky J, van Melle G and Regli F: The Lausanne stroke registry: analysis of 1,000 consecutive patients with first stroke. Stroke 19: 1083-1092, 1988.

3. Kwakkel G, Kollen BJ, van der Grond J and Prevo AJ: Probability of regaining dexterity in the flaccid upper limb: impact of severity of paresis and time since onset in acute stroke. Stroke 34: 2181-2186, 2003.

4. Lai SM, Studenski S, Duncan PW and Perera S: Persisting consequences of stroke measured by the Stroke Impact Scale. Stroke 33: 1840-1844, 2002.

5. Zinn S, Dudley TK, Bosworth HB, Hoenig HM, Duncan PW and Horner RD: The effect of poststroke cognitive impairment on rehabilitation process and functional outcome. Arch Phys Med Rehabil 85: 1084-1090, 2004.

6. Samsa GP and Matchar DB: How strong is the relationship between functional status and quality of life among persons with stroke? J Rehabil Res Dev 41: 279-282, 2004.

7. Bach-y-Rita P: Brain plasticity as a basis for recovery of function in humans. Neuropsychologia 28: 547-554, 1990.

8. Johansson BB: Brain plasticity and stroke rehabilitation. The Willis lecture. Stroke 31: 223-230, 2000.

9. Lee RG and van Donkelaar P: Mechanisms underlying functional recovery following stroke. Can J Neurol Sci 22: 257-263, 1995.
10. Seil FJ: Recovery and repair issues after stroke from the scientific perspective. Curr Opin Neurol 10: 49-51, 1997.

11. Steinberg BA and Augustine JR: Behavioral, anatomical, and physiological aspects of recovery of motor function following stroke. Brain Res Brain Res Rev 25: 125-132, 1997.

12. Weiller C: Imaging recovery from stroke. Exp Brain Res 123 : 13-17, 1998.

13. Dijkhuizen RM, Ren J, Mandeville JB, et al: Functional magnetic resonance imaging of reorganization in rat brain after stroke. Proc Natl Acad Sci USA 98: 12766-12771, 2001.

14. Schaechter JD, Kraft E, Hilliard TS, et al: Motor recovery and cortical reorganization after constraint-induced movement therapy in stroke patients: a preliminary study. Neurorehabil Neural Repair 16: 326-338, 2002.

15. Dijkhuizen RM, Singhal AB, Mandeville JB, et al: Correlation between brain reorganization, ischemic damage, and neurologic status after transient focal cerebral ischemia in rats: a functional magnetic resonance imaging study. J Neurosci 23: 510-517, 2003.

16. Jenkins WM and Merzenich MM: Reorganization of neocortical representations after brain injury: a neurophysiological model of the bases of recovery from stroke. Prog Brain Res 71: 249-266, 1987.

17. Nudo RJ and Milliken GW: Reorganization of movement representations in primary motor cortex following focal ischemic infarcts in adult squirrel monkeys. J Neurophysiol 75: 2144-2149, 1996.

18. Indredavik B, Slordahl SA, Bakke F, Rokseth R and Haheim LL: Stroke unit treatment. Long-term effects. Stroke 28: 1861-1866, 1997.

19. Pomeroy VM and Tallis RC: Need to focus research in stroke rehabilitation. Lancet 355: 836-837, 2000.

20. Duncan PW, Goldstein LB, Matchar D, Divine GW and Feussner J: Measurement of motor recovery after stroke. Outcome assessment and sample size requirements. Stroke 23: 1084-1089, 1992.

21. Hendricks HT, van Limbeek J, Geurts AC and Zwarts MJ: Motor recovery after stroke: a systematic review of the literature. Arch Phys Med Rehabil 83: 1629-1637, 2002.

22. Binkofski F and Seitz RJ: Modulation of the BOLD-response in early recovery from sensorimotor stroke. Neurology 63: 1223-1229, 2004.

23. Carmichael ST, Tatsukawa K, Katsman D, Tsuyuguchi N and Kornblum HI: Evolution of diaschisis in a focal stroke model. Stroke 35: 758-763, 2004.

24. Taub E, Miller NE, Novack TA, et al: Technique to improve chronic motor deficit after stroke. Arch Phys Med Rehabil 74: 347-354, 1993.

25. Miltner WH, Bauder H, Sommer M, Dettmers C and Taub E: Effects of constraint-induced movement therapy on patients with chronic motor deficits after stroke: a replication. Stroke 30: 586-592, 1999.

26. Van der Lee JH, Wagenaar RC, Lankhorst GJ, Vogelaar TW, Deville WL and Bouter LM: Forced use of the upper extremity in chronic stroke patients: results from a single-blind randomized clinical trial. Stroke 30: 2369-2375, 1999.

27. Liepert J, Bauder H, Wolfgang HR, Miltner WH, Taub E and Weiller C: Treatment-induced cortical reorganization after stroke in humans. Stroke 31: 1210-1216, 2000.

28. Lum PS, Burgar CG, Shor PC, Majmundar M and van der Loos M: Robot-assisted movement training compared with conventional therapy techniques for the rehabilitation of upper-limb motor function after stroke. Arch Phys Med Rehabil 83: 952-959, 2002.

29. Fasoli SE, Krebs HI, Stein J, Frontera WR and Hogan N: Effects of robotic therapy on motor impairment and recovery in chronic stroke. Arch Phys Med Rehabil 84: 477-482, 2003.

30. Fasoli SE, Krebs HI, Stein J, Frontera WR, Hughes R and Hogan N: Robotic therapy for chronic motor impairments after stroke: follow-up results. Arch Phys Med Rehabil 85: 1106-1111, 2004.

31. Taub E, Uswatte G, King DK, Morris D, Crago JE and Chatterjee A: A placebo-controlled trial of constraint-induced movement therapy for upper extremity after stroke. Stroke 37: 1045-1049, 2006.

32. Duncan PW, Zorowitz R, Bates B, et al: Management of Adult Stroke Rehabilitation Care: a clinical practice guideline. Stroke 36: e100-e143, 2005.

33. Eliassen JC, Boespflug EL, Lamy M, Allendorfer J, Chu WJ and Szaflarski JP: Brain-mapping techniques for evaluating poststroke recovery and rehabilitation: a review. Top Stroke Rehabil 15: 427-450, 2008. 
34. Hillebrand A, Singh KD, Holliday IE, Furlong PL and Barnes GR A new approach to neuroimaging with magnetoencephalography. Hum Brain Mapp 25: 199-211, 2005.

35. Lauritzen M: Reading vascular changes in brain imaging: is dendritic calcium the key? Nat Rev Neurosci 6: 77-85, 2005.

36. Costafreda SG, Fu CH, Lee L, Everitt B, Brammer MJ and David AS: A systematic review and quantitative appraisal of fMRI studies of verbal fluency: role of the left inferior frontal gyrus. Hum Brain Mapp 27: 799-810, 2006.

37. Debener S, Ullsperger M, Siegel M and Engel AK: Single-trial EEG-fMRI reveals the dynamics of cognitive function. Trends Cogn Sci 10: 558-563, 2006.

38. Norris DG: Principles of magnetic resonance assessment of brain function. J Magn Reson Imaging 23: 794-807, 2006.

39. Otte A and Halsband U: Brain imaging tools in neurosciences. J Physiol Paris 99: 281-292, 2006.

40. Stern JM: Simultaneous electroencephalography and functional magnetic resonance imaging applied to epilepsy. Epilepsy Behav 8: 683-692, 2006

41. Van Eimeren T and Siebner HR: An update on functional neuroimaging of parkinsonism and dystonia. Curr Opin Neurol 19: 412-419, 2006

42. Shibasaki H: Human brain mapping: hemodynamic response and electrophysiology. Clin Neurophysiol 119: 731-743, 2008.

43. Horwitz B and Poeppel D: How can EEG/MEG and fMRI/PET data be combined? Hum Brain Mapp 17: 1-3, 2002.

44. Sammer G, Blecker C, Gebhardt H, Kirsch P, Stark R and Vaitl D: Acquisition of typical EEG waveforms during fMRI: SSVEP, LRP, and frontal theta. Neuroimage 24: 1012-1024, 2005.

45. Horovitz SG, Fukunaga M, de Zwart JA, et al: Low frequency BOLD fluctuations during resting wakefulness and light sleep: a simultaneous EEG-fMRI study. Hum Brain Mapp 29: 671-682, 2008.

46. Kramer MA, Eden UT, Lepage KQ, Kolaczyk ED, Bianchi MT and Cash SS: Emergence of persistent networks in long-term intracranial EEG recordings. J Neurosci 31: 15757-15767, 2011.

47. Ritter P, Becker R, Graefe C and Villringer A: Evaluating gradient artifact correction of EEG data acquired simultaneously with fMRI. Magn Reson Imaging 25: 923-932, 2007.

48. Jager L, Werhahn KJ, Hoffmann A, et al: Focal epileptiform activity in the brain: detection with spike-related functional MR imaging - preliminary results. Radiology 223: 860-869, 2002.

49. Lev $\mathrm{MH}$ and Grant PE: MEG versus BOLD MR imaging: functional imaging, the next generation? Am J Neuroradiol 21: 1369-1370, 2000.

50. Ogawa S, Tank DW, Menon R, et al: Intrinsic signal changes accompanying sensory stimulation: functional brain mapping with magnetic resonance imaging. Proc Natl Acad Sci USA 89: 5951-5955, 1992

51. Frahm J, Bruhn H, Merboldt KD and Hanicke W: Dynamic MR imaging of human brain oxygenation during rest and photic stimulation. J Magn Reson Imaging 2: 501-505, 1992.

52. Kim SG, Ashe J, Georgopoulos AP, et al: Functional imaging of human motor cortex at high magnetic field. J Neurophysiol 69: 297-302, 1993

53. Kwong KK, Belliveau JW, Chesler DA, et al: Dynamic magnetic resonance imaging of human brain activity during primary sensory stimulation. Proc Natl Acad Sci USA 89: 5675-5679, 1992.

54. Bandettini PA, Wong EC, Hinks RS, Tikofsky RS and Hyde JS Time course EPI of human brain function during task activation. Magn Reson Med 25: 390-397, 1992.

55. Mattay VS, Frank JA, Santha AK, et al: Whole-brain functional mapping with isotropic MR imaging. Radiology 201: 399-404, 1996.

56. Meyer CH, Hu BS, Nishimura DG and Macovski A: Fast spiral coronary artery imaging. Magn Reson Med 28: 202-213, 1992.

57. Noll DC, Cohen JD, Meyer CH and Schneider W: Spiral K-space MR imaging of cortical activation. J Magn Reson Imaging 5: 49-56, 1995.

58. Glover GH and Lee AT: Motion artifacts in fMRI: comparison of 2DFT with PR and spiral scan methods. Magn Reson Med 33: 624-635, 1995

59. Yang Y, Glover GH, van Gelderen P, et al: Fast 3D functional magnetic resonance imaging at $1.5 \mathrm{~T}$ with spiral acquisition. Magn Reson Med 36: 620-626, 1996.

60. Liu G, Sobering G, Duyn J and Moonen CT: A functional MRI technique combining principles of echo-shifting with a train of observations (PRESTO). Magn Reson Med 30: 764-768, 1993.
61. Ramsey NF, Kirkby BS, van Gelderen P, et al: Functional mapping of human sensorimotor cortex with 3D BOLD fMRI correlates highly with $\mathrm{H} 2(15) \mathrm{O}$ PET rCBF. J Cereb Blood Flow Metab 16: 755-764, 1996

62. Callicott JH, Ramsey NF, Tallent K, et al: Functional magnetic resonance imaging brain mapping in psychiatry: methodological issues illustrated in a study of working memory in schizophrenia. Neuropsychopharmacology 18: 186-196, 1998.

63. Buckner RL, Bandettini PA, O'Craven KM, et al: Detection of cortical activation during averaged single trials of a cognitive task using functional magnetic resonance imaging. Proc Natl Acad Sci USA 93: 14878-14883, 1996.

64. Zarahn E, Aguirre G and D'Esposito M: A trial-based experimental design for fMRI. Neuroimage 6: 122-138, 1997.

65. Friston KJ, Fletcher P, Josephs O, Holmes A, Rugg MD and Turner R: Event-related fMRI: characterizing differential responses. Neuroimage 7: 30-40, 1998.

66. Josephs $\mathrm{O}$ and Henson RN: Event-related functional magnetic resonance imaging: modelling, inference and optimization. Philos Trans R Soc Lond B Biol Sci 354: 1215-1228, 1999.

67. Rosen BR, Buckner RL and Dale AM: Event-related functional MRI: past, present, and future. Proc Natl Acad Sci USA 95: 773-780, 1998 .

68. Van Meer MP, van der Marel K, Wang K, et al: Recovery of sensorimotor function after experimental stroke correlates with restoration of resting-state interhemispheric functional connectivity. J Neurosci 30: 3964-3972, 2010.

69. Detre JA and Alsop DC: Perfusion magnetic resonance imaging with continuous arterial spin labeling: methods and clinical applications in the central nervous system. Eur J Radiol 30: 115-124, 1999.

70. Edelman R, Siewert B, Darby D, et al: Qualitative mapping of cerebral blood flow and functional localization with echoplanar MR imaging and signal targeting with alternating radio frequency. Radiology 192: 513-520, 1994.

71. Aslop D and Detre J: Reduced transiti-time sensitivity in noninvasive magnetic resonance imaging of human cerebral blood flow. J Cereb Blood Flow Metab 16: 1236-1249, 1996.

72. Kim S: Quantification of relative cerebral blood flow change by flowsensitive alternating inversion recovery (FAIR) technique: application to functional mapping. Magn Reson Med 34: 293-301, 1995.

73. Ye FQ, Smith AM, Yang Y, et al: Quantitation of regional cerebral blood flow increases during motor activation: a steadystate arterial spin tagging study. Neuroimage 6: 104-112, 1997.

74. Aguirre GK, Detre JA, Zarahn E and Alsop DC: Experimental design and the relative sensitivity of BOLD and perfusion fMRI. Neuroimage 15: 488-500, 2002.

75. Yang Y,Engelien W, Pan H, Xu S, Silbersweig DA and Stern E: A CBF-based event-related brain activation paradigm: characterization of impulse-response function and comparison to BOLD. Neuroimage 12: 287-297, 2000

76. Liu HL, Pu Y, Nickerson LD, Liu Y, Fox PT and Gao JH: Comparison of the temporal response in perfusion and BOLDbased event-related functional MRI. Magn Reson Med 43: 768-772, 2000.

77. Kim SG, Tsekos NV and Ashe J: Multi-slice perfusion-based functional MRI using the FAIR technique: comparison of CBF and BOLD effects. NMR Biomed 10: 191-196, 1997.

78. Luh WM, Wong EC, Bandettini PA, Ward BD and Hyde JS: Comparison of simultaneously measured perfusion and BOLD signal increases during brain activation with $\mathrm{T}(1)$-based tissue identification. Magn Reson Med 44: 137-143, 2000.

79. Yongbi MN, Fera F, Mattay VS, Frank JA and Duyn JH: Simultaneous BOLD/perfusion measurement using dual-echo FAIR and UNFAIR: sequence comparison at 1.5T and 3.0T. Magn Reson Imaging 19: 1159-1165, 2001.

80. Baron JC, Cohen LG, Cramer SC, et al: Neuroimaging in stroke recovery: a position paper from the First International Workshop on Neuroimaging and Stroke Recovery. Cerebrovasc Dis 18: 260-267, 2004.

81. Cramer SC and Bastings EP: Mapping clinically relevant plasticity after stroke. Neuropharmacology 39: 842-851, 2000.

82. Chollet F, DiPiero V, Wise RJ, Brooks DJ, Dolan RJ and Frackowiak RS: The functional anatomy of motor recovery after stroke in humans: a study with positron emission tomography. Ann Neurol 29: 63-71, 1991.

83. Weiller C, Chollet F, Friston KJ, Wise RJ and Frackowiak RS: Functional reorganization of the brain in recovery from striatocapsular infarction in man. Ann Neurol 31: 463-472, 1992. 
84. Weiller C, Ramsay SC, Wise RJ, Friston KJ and Frackowiak RS Individual patterns of functional reorganization in the human cerebral cortex after capsular infarction. Ann Neurol 33: 181-189, 1993.

85. Seitz RJ, Hoflich P, Binkofski F, Tellmann L, Herzog H and Freund HJ: Role of the premotor cortex in recovery from middle cerebral artery infarction. Arch Neurol 55: 1081-1088, 1998.

86. Honda M, Nagamine T, Fukuyama H, Yonekura Y, Kimura J and Shibasaki H: Movement-related cortical potentials and regional cerebral blood flow change in patients with stroke after motor recovery. J Neurol Sci 146: 117-126, 1997.

87. Nelles G, Spiekermann G, Jueptner M, et al: Reorganization of sensory and motor systems in hemiplegic stroke patients. A positron emission tomography study. Stroke 30: 1510-1516, 1999.

88. Nelles G, Spiekramann G, Jueptner M, et al: Evolution of functional reorganization in hemiplegic stroke: a serial positron emission tomographic activation study. Ann Neurol 46: 901-909, 1999.

89. Pizzamiglio L, Perani D, Cappa SF, et al: Recovery of neglect after right hemispheric damage: $\mathrm{H} 2(15) \mathrm{O}$ positron emission tomographic activation study. Arch Neurol 55: 561-568, 1998.

90. Weiller C, Isensee C, Rijntjes M, et al: Recovery from Wernicke's aphasia: a positron emission tomographic study. Ann Neurol 37: 723-732, 1995.

91. Buckner RL, Corbetta M, Schatz J, Raichle ME and Petersen SE: Preserved speech abilities and compensation following prefrontal damage. Proc Natl Acad Sci USA 93: 1249-1253, 1996

92. Heiss WD, Kessler J, Thiel A, Ghaemi M and Karbe H: Differential capacity of left and right hemispheric areas for compensation of poststroke aphasia. Ann Neurol 45: 430-438, 1999.

93. Nelles G, Jentzen W, Jueptner M, Muller S and Diener HC: Arm training induced brain plasticity in stroke studied with serial positron emission tomography. Neuroimage 13: 1146-1154, 2001.

94. Schaechter JD, Moore CI, Connell BD, Rosen BR and Dijkhuizen RM: Structural and functional plasticity in the somatosensory cortex of chronic stroke patients. Brain 129: 2722-2733, 2006.

95. Mintzopoulos D, Khanicheh A, Konstas AA, et al: Functional MRI of rehabilitation in chronic stroke patients using novel MR-compatible hand robots. Open Neuroimag J 2: 94-101, 2008.

96. Mintzopoulos D, Astrakas LG, Khanicheh A, et al: Connectivity alterations assessed by combining fMRI and MR-compatible hand robots in chronic stroke. Neuroimage 47 (Suppl 2): T90-T97, 2009.

97. Carey LM and Seitz RJ: Functional neuroimaging in stroke recovery and neurorehabilitation: conceptual issues and perspectives. Int J Stroke 2: 245-264, 2007.

98. Cramer SC, Finklestein SP, Schaechter JD, Bush G and Rosen BR: Activation of distinct motor cortex regions during ipsilateral and contralateral finger movements. J Neurophysiol 81: 383-387, 1999

99. Cao Y, Vikingstad EM, Huttenlocher PR, Towle VL and Levin DN: Functional magnetic resonance studies of the reorganization of the human hand sensorimotor area after unilateral brain injury in the perinatal period. Proc Natl Acad Sci USA 91: 9612-9616, 1994.

100. Cramer SC, Nelles G, Benson RR, et al: A functional MRI study of subjects recovered from hemiparetic stroke. Stroke 28: 2518-2527, 1997.

101. Cao Y, D'Olhaberriague L, Vikingstad EM, Levine SR and Welch KM: Pilot study of functional MRI to assess cerebral activation of motor function after poststroke hemiparesis. Stroke 29: 112-122, 1998.

102. Thulborn KR, Carpenter PA and Just MA: Plasticity of language-related brain function during recovery from stroke. Stroke 30: 749-754, 1999.

103. Cao Y, Vikingstad EM, George KP, Johnson AF and Welch KM: Cortical language activation in stroke patients recovering from aphasia with functional MRI. Stroke 30: 2331-2340, 1999.

104. Marshall RS, Perera GM, Lazar RM, Krakauer JW, Constantine RC and DeLaPaz RL: Evolution of cortical activation during recovery from corticospinal tract infarction. Stroke 31: 656-661, 2000.

105. Pineiro R, Pendlebury S, Johansen-Berg H and Matthews PM: Functional MRI detects posterior shifts in primary sensorimotor cortex activation after stroke: evidence of local adaptive reorganization? Stroke 32: 1134-1139, 2001.
106. Johansen-Berg H, Dawes H, Guy C, Smith SM, Wade DT and Matthews PM: Correlation between motor improvements and altered fMRI activity after rehabilitative therapy. Brain 125: 2731-2742, 2002.

107. Johansen-Berg H, Rushworth MF, Bogdanovic MD, Kischka U, Wimalaratna S and Matthews PM: The role of ipsilateral premotor cortex in hand movement after stroke. Proc Natl Acad Sci USA 99: 14518-14523, 2002.

108. Dong Y, Dobkin BH, Cen SY, Wu AD and Winstein CJ: Motor cortex activation during treatment may predict therapeutic gains in paretic hand function after stroke. Stroke 37: 1552-1555, 2006.

109. Miyai I, Suzuki M, Hatakenaka M and Kubota K: Effect of body weight support on cortical activation during gait in patients with stroke. Exp Brain Res 169: 85-91, 2006.

110. Miyai I, Yagura H, Hatakenaka M, Oda I, Konishi I and Kubota K: Longitudinal optical imaging study for locomotor recovery after stroke. Stroke 34: 2866-2870, 2003

111. Miyai I, Yagura H, Oda I, et al: Premotor cortex is involved in restoration of gait in stroke. Ann Neurol 52: 188-194, 2002.

112. Kopp B, Kunkel A, Muhlnickel W, Villringer K, Taub E and Flor H: Plasticity in the motor system related to therapy-induced improvement of movement after stroke. Neuroreport 10: 807-810, 1999.

113. Murata Y, Sakatani K, Hoshino T, et al: Effects of cerebral ischemia on evoked cerebral blood oxygenation responses and BOLD contrast functional MRI in stroke patients. Stroke 37: 2514-2520, 2006.

114. Hamzei F, Knab R, Weiller C and Rother J: The influence of extra- and intracranial artery disease on the BOLD signal in FMRI. Neuroimage 20: 1393-1399, 2003

115. Di Lazzaro V, Pilato F, Dileone M, et al: Modulating cortical excitability in acute stroke: a repetitive TMS study. Clin Neurophysiol 119: 715-723, 2008.

116. Bernad DM and Doyon J: The role of noninvasive techniques in stroke therapy. Int J Biomed Imaging 2008: 672582, 2008.

117. Barnes MP: Rehabilitation after traumatic brain injury. Br Med Bull 55: 927-943, 1999

118. Deutsch JE, Latonio J, Burdea G and Boian R: Rehabilitation of musculoskeletal injuries using the Rutgers ankle haptic interface: three case reports. 2001 Eurohaptics Conference, Birmingham UK. Eurohaptics 2001: 11-16, 2001.

119. Jack D, Boian R, Merians A, et al: Virtual reality-enhanced stroke rehabilitation. IEEE Trans Neural Syst Rehabil Eng 9: 308-318, 2001

120. Lum P, Reinkensmeyer D, Mahoney R, Rymer WZ and Burgar C: Robotic devices for movement therapy after stroke: current status and challenges to clinical acceptance. Top Stroke Rehabil 8: 40-53, 2002.

121. Schaechter JD: Motor rehabilitation and brain plasticity after hemiparetic stroke. Prog Neurobiol 73: 61-72, 2004.

122. Fisch A, Mavroidis C, Melli-Huber J and Bar-Cohen Y: Haptic devices for virtual reality, telepresence, and human-assistive robotics. In: Biologically-Inspired Intelligent Robots. Bar-Cohen Y and Breazeal CL (eds). SPIE Press, pp73-101, 2003.

123. Huang VS and Krakauer JW: Robotic neurorehabilitation: a computational motor learning perspective. J Neuroeng Rehabil 6: 5,2009

124. Tsekos N, Khanicheh A, Christoforou E and Mavroidis C: Magnetic resonance-compatible robotic and mechatronics systems for image-guided interventions and rehabilitation: a review study. Annu Rev Biomed Eng 9: 351-387, 2007.

125. Aisen ML, Krebs HI, Hogan N, McDowell F and Volpe BT: The effect of robot-assisted therapy and rehabilitative training on motor recovery following stroke. Arch Neurol 54: 443-446, 1997.

126. Volpe BT, Krebs HI, Hogan N, Edelstein OL, Diels C and Aisen M: A novel approach to stroke rehabilitation: robot-aided sensorimotor stimulation. Neurology 54: 1938-1944, 2000.

127. Volpe BT, Krebs HI, Hogan N, Edelsteinn L, Diels CM and Aisen ML: Robot training enhanced motor outcome in patients with stroke maintained over 3 years. Neurology 53: 1874-1876, 1999.

128. Volpe BT, Krebs HI and Hogan N: Is robot-aided sensorimotor training in stroke rehabilitation a realistic option? Curr Opin Neurol 14: 745-752, 2001

129. Volpe BT, Ferraro M, Lynch D, et al: Robotics and other devices in the treatment of patients recovering from stroke. Curr Neurol Neurosci Rep 5: 465-470, 2005.

130. Volpe BT, Ferraro M, Krebs HI and Hogan N: Robotics in the rehabilitation treatment of patients with stroke. Curr Atheroscler Rep 4: 270-276, 2002. 
131. Ferraro M, Palazzolo JJ, Krol J, Krebs HI, Hogan N and Volpe BT: Robot-aided sensorimotor arm training improves outcome in patients with chronic stroke. Neurology 61: 1604-1607, 2003.

132. Daly JJ, Hogan N, Perepezko EM, et al: Response to upper-limb robotics and functional neuromuscular stimulation following stroke. J Rehabil Res Dev 42: 723-736, 2005.

133. Macclellan LR, Bradham DD, Whitall J, et al: Robotic upperlimb neurorehabilitation in chronic stroke patients. J Rehabil Res Dev 42: 717-722, 2005.

134. Finley MA, Fasoli SE, Dipietro L, et al: Short-duration robotic therapy in stroke patients with severe upper-limb motor impairment. J Rehabil Res Dev 42: 683-692, 2005.

135. Prange GB, Jannink MJ, Groothuis-Oudshoorn CG, Hermens HJ and Ijzerman MJ: Systematic review of the effect of robot-aided therapy on recovery of the hemiparetic arm after stroke. J Rehabil Res Dev 43: 171-184, 2006.

136. Brewer BR, McDowell SK and Worthen-Chaudhari LC: Poststroke upper extremity rehabilitation: a review of robotic systems and clinical results. Top Stroke Rehabil 14: 22-44, 2007.

137. Patton J, Dawe G, Scharver C, Mussa-Ivaldi F and Kenyon R: Robotics and virtual reality: a perfect marriage for motor control research and rehabilitation. Assist Technol 18: 181-195, 2006.

138. Cramer SC, Nelles G, Schaechter JD, Kaplan JD, Finklestein SP and Rosen BR: A functional MRI study of three motor tasks in the evaluation of stroke recovery. Neurorehabil Neural Repair 15: 1-8, 2001.

139. Hidler J, Hodics T, Xu B, Dobkin B and Cohen LG: MR compatible force sensing system for real-time monitoring of wrist moments during fMRI testing. J Neurosci Methods 155: 300-307, 2006.

140. Liu JZ, Dai TH, Elster TH, Sahgal V, Brown RW and Yue GH: Simultaneous measurement of human joint force, surface electromyograms, and functional MRI-measured brain activation. J Neurosci Methods 101: 49-57, 2000.

141. Riener R, Villgrattner T, Kleiser R, Nef T and Kollias S: fMRIcompatible electromagnetic haptic interface. 27th Annual International Conference of the IEEE, Shanghai, China IEEE-EMBS 2005: 7024-7027, 2005.

142. Diedrichsen J, Hashambhoy Y, Rane T and Shadmehr R: Neural correlates of reach errors. J Neurosci 25: 9919-9931, 2005.

143. Gassert R, Dovat L, Lambercy O, et al: A 2-DOF fMRI compatible haptic interface to investigate the neural control of arm movements. IEEE International Conference on Robotics and Automation, Orlando, FL. ICRA 2006: 3825-3831, 2006.

144. Liepert J, Miltner WH, Bauder H, et al: Motor cortex plasticity during constraint-induced movement therapy in stroke patients. Neurosci Lett 250: 5-8, 1998.

145. Khanicheh A, Muto A, Triantafyllou C, et al: fMRI-compatible rehabilitation hand device. J Neuroengineering Rehabl 3: 24-35, 2006.

146. Khanicheh A, Muto A, Triantafyllou C, Astrakas LG, Mavroidis C and Tzika A: MR compatible ERF-based robotic device for hand rehabilitation after stroke. Proc Intl Soc Mag Reson Med 13: 1110, 2005

147. Tzika A, Khanicheh A, Muto A, Triantafyllou C, Astrakas LG and Mavroidis C: Novel rehabilitation hand robots and fMRI in stroke. Eur Radiol 16 (Suppl 1): 183, 2006.

148. Khanicheh A, Mintzopoulos D, Weinberg B, Tzika AA and Mavroidis C: MR CHIROD v.2: a fMRI compatible mechatronic hand rehabilitation device. IEEE 10th Internationa Conference on Rehabilitation Robotics IEEE, Noodwijk, The Netherlands. ICRA 2007: pp883-889, 2007.

149. Khanicheh A, Mintzopoulos D, Weinberg B, Tzika AA and Mavroidis C: MR CHIROD v.2: magnetic resonance compatible smart hand rehabilitation device for brain imaging. IEEE Trans Neural Syst Rehabil Eng 16: 91-98, 2008.

150. Siekierka EM, Eng K, Bassetti C, et al: New technologies and concepts for rehabilitation in the acute phase of stroke: a collaborative matrix. Neurodegener Dis 4: 57-69, 2007.
151. Colombo R, Pisano F, Mazzone A, et al: Design strategies to improve patient motivation during robot-aided rehabilitation. $\mathrm{J}$ Neuroeng Rehabil 4: 3, 2007.

152. Hesse S, Schulte-Tigges G, Konrad M, Bardeleben A and Werner C: Robot-assisted arm trainer for the passive and active practice of bilateral forearm and wrist movements in hemiparetic subjects. Arch Phys Med Rehabil 84: 915-920, 2003.

153. Khanicheh A, Mintzopoulos D, Weinberg B, Tzika AA and Mavroidis C: Evaluation of electrorheological fluid dampers for applications at 3-Tesla MRI environment. IEEE/ASME Trans Mechatronics 13: 286-294, 2008.

154. Kim YR, Huang IJ, Lee SR, et al: Measurements of BOLD/CBV ratio show altered fMRI hemodynamics during stroke recovery in rats. J Cereb Blood Flow Metab 25: 820-829, 2005.

155. Triantafyllou C, Hoge RD, Krueger G, et al: Comparison of physiological noise at $1.5 \mathrm{~T}, 3 \mathrm{~T}$ and $7 \mathrm{~T}$ and optimization of fMRI acquisition parameters. Neuroimage 26: 243-250, 2005.

156. Heidemann RM, Griswold MA, Seiberlich N, et al: Direct parallel image reconstructions for spiral trajectories using GRAPPA. Magn Reson Med 56: 317-326, 2006

157. Setsompop K, Wald LL, Alagappan V, et al: Parallel RF transmission with eight channels at 3 Tesla. Magn Reson Med 56: 1163-1171, 2006.

158. Alagappan V, Nistler J, Adalsteinsson E, et al: Degenerate mode band-pass birdcage coil for accelerated parallel excitation. Magn Reson Med 57: 1148-1158, 2007.

159. Mintzopoulos D, Khanicheh A, Mavroidis C, Astrakas LG, Zurakowski D and Tzika AA: On-line brain mapping using fMRI and a Magnetic Resonance Compatible Hand-Induced Robotic Device (MR_CHIROD). Proc Intl Soc Mag Reson Med 15: 3330, 2007.

160. Mintzopoulos D, Astrakas LG, Wiggins GC, Wald LL, Rosen BR and Tzika AA: Improved BOLD detection at 3T using high-resolution GRAPPA EPI FMRI. Proc Intl Soc Mag Reson Med 16: 2375, 2008.

161. Mintzopoulos D, Astrakas LG, Koroshetz W, Moskowitz MA, Rosen BR and Tzika AA: fMRI of rehabilitation in chronic stroke using MR-compatible robots. Proc Intl Soc Mag Reson Med 16: 3285, 2008.

162. Mintzopoulos D, Astrakas LG, Hiroki M, et al: fMRI using GRAPPA EPI with high spatial resolution improves BOLD signal detection at 3T. Open Magn Resonance J 2: 57-70, 2009.

163. Crafton KR, Mark AN and Cramer SC: Improved understanding of cortical injury by incorporating measures of functional anatomy. Brain 126: 1650-1659, 2003.

164. Stinear CM, Barber PA, Smale PR, Coxon JP, Fleming MK and Byblow WD: Functional potential in chronic stroke patients depends on corticospinal tract integrity. Brain 130: 170-180, 2007.

165. Binkofski F, Seitz RJ, Arnold S, Classen J, Benecke R and Freund HJ: Thalamic metbolism and corticospinal tract integrity determine motor recovery in stroke. Ann Neurol 39: 460-470, 1996.

166. Ward NS, Newton JM, Swayne OB, et al: Motor system activation after subcortical stroke depends on corticospinal system integrity. Brain 129: 809-819, 2006.

167. Butefisch CM, Kleiser R and Seitz RJ: Post-lesional cerebral reorganisation: evidence from functional neuroimaging and transcranial magnetic stimulation. J Physiol Paris 99: 437-454, 2006.

168. Schaechter JD, Perdue KL and Wang R: Structural damage to the corticospinal tract correlates with bilateral sensorimotor cortex reorganization in stroke patients. Neuroimage 39: 1370-1382, 2008 . 